\title{
Acute kidney injury (AKI) after a single intravenous zoledronic acid administration in patients with osteoporosis
}

\author{
C. Cipriani, C. Clementelli, V. Fassino, R. Manai, V. Danese, V. Cecchetti, F. Ferrone, J. Pepe, S Minisola \\ Department of Internal Medicine and Medical Disciplines, "Sapienza" University of Rome
}

Introduction

Safety data on zoledronic acid (ZA) administration for osteoporosis suggest potential kidney toxicity; indeed, it is not indicated in patients with impaired renal function. Patients' clinical monitoring usually includes glomerular filtration rate (GFR) evaluation; no study addressed the issue of the early kidney injury.

We evaluated the early (within 3 months) effect of iv ZA on renal function defining the potential role of AKI biomarkers in unveiling subtle damage.

\section{Patients and Methods}

Five $\mathrm{mg}$ i.v. ZA infusion was administered for the first time to 23 patients with osteoporosis and normal renal function

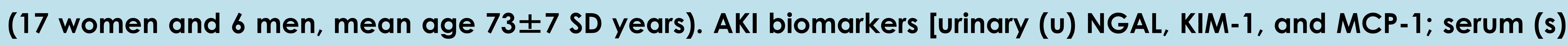
MCP-1 and IL-18] were assessed at baseline and at day (d) 2 and 30 after administration. Serum calcium (sCa), Creatinine clearance $(\mathrm{CrCl})$, parathyroid hormone (PTH), plasma C-terminal FGF-23, sKlotho, calcium excretion (CaEx) and renal threshold phosphate concentration/GFR (TmPO 4 /GFR) were also measured.

Results

No significant changes in mean levels of urinary markers were detected. Mean values of slL-18 and SMCP-1

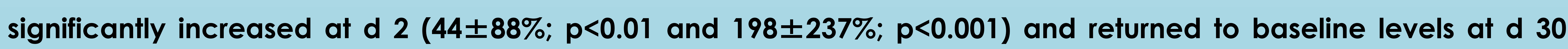
(Figure 1). There was a significant decrease in $\mathrm{sCa}$ at $\mathrm{d} 2(-4.1 \pm 2.8 \% ; \mathrm{p}<0.01)$ and $\mathrm{d} 30(-3.9 \pm 4 \% ; \mathrm{p}<0.001)$. Mean $\mathrm{CrCl}$ values did not significantly change at $d 30$. Serum PTH significantly increase by $29.8 \pm 37.7 \%$ at $d 2(p<0.05)$ and $79.8 \pm 95.8 \%$ at $d 30$ ( $p<0.001$ ) (Figure 2). There was a $28 \pm 59 \%$ and $26 \pm 43 \%$ decrease in CaEx at $d 2(p<0.05)$ and $d$ $30(p<0.01$ ), respectively (Figure 2$) . \mathrm{TmPO}_{4} /$ GFR significantly decreased at $d 2$ and $d 30(-8.6 \pm 15.9 \%, p<0.05$ and $11.3 \pm 13.5 \%, p<0.001$ ) (Figure 2). We observed no difference in mean levels of plasma C-terminal FGF-23 and sKlotho at any time.

Figure 1. Mean values of serum MCP-1 and serum IL-18 percent basal difference at any time point

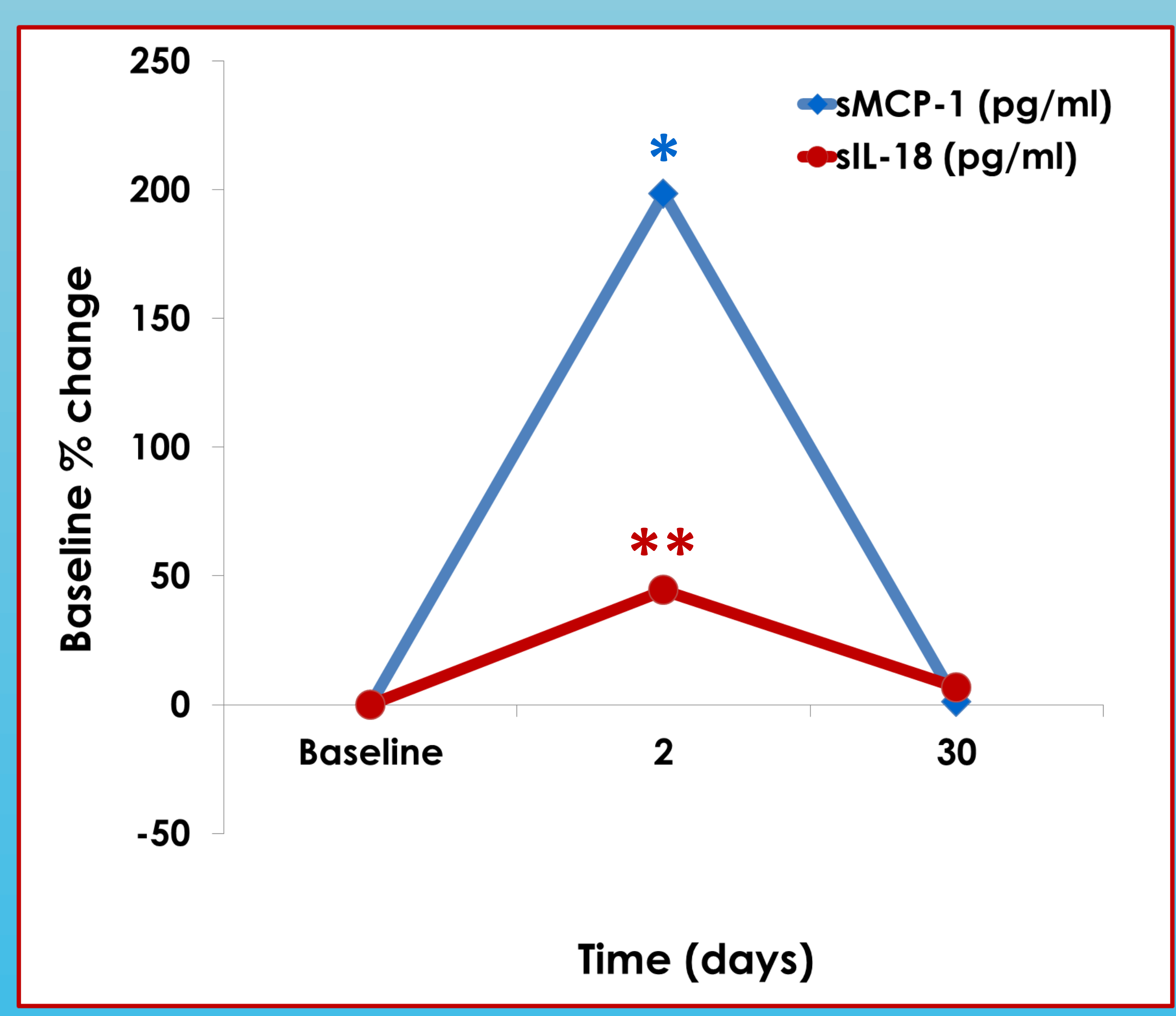

"p $<0.001 ; "{ }^{*} p<0.01$
Figure 2. Mean values of serum Calcium, PTH, calcium excretion and $\mathrm{ImPO}_{4} /$ GFR percent basal difference at any time point

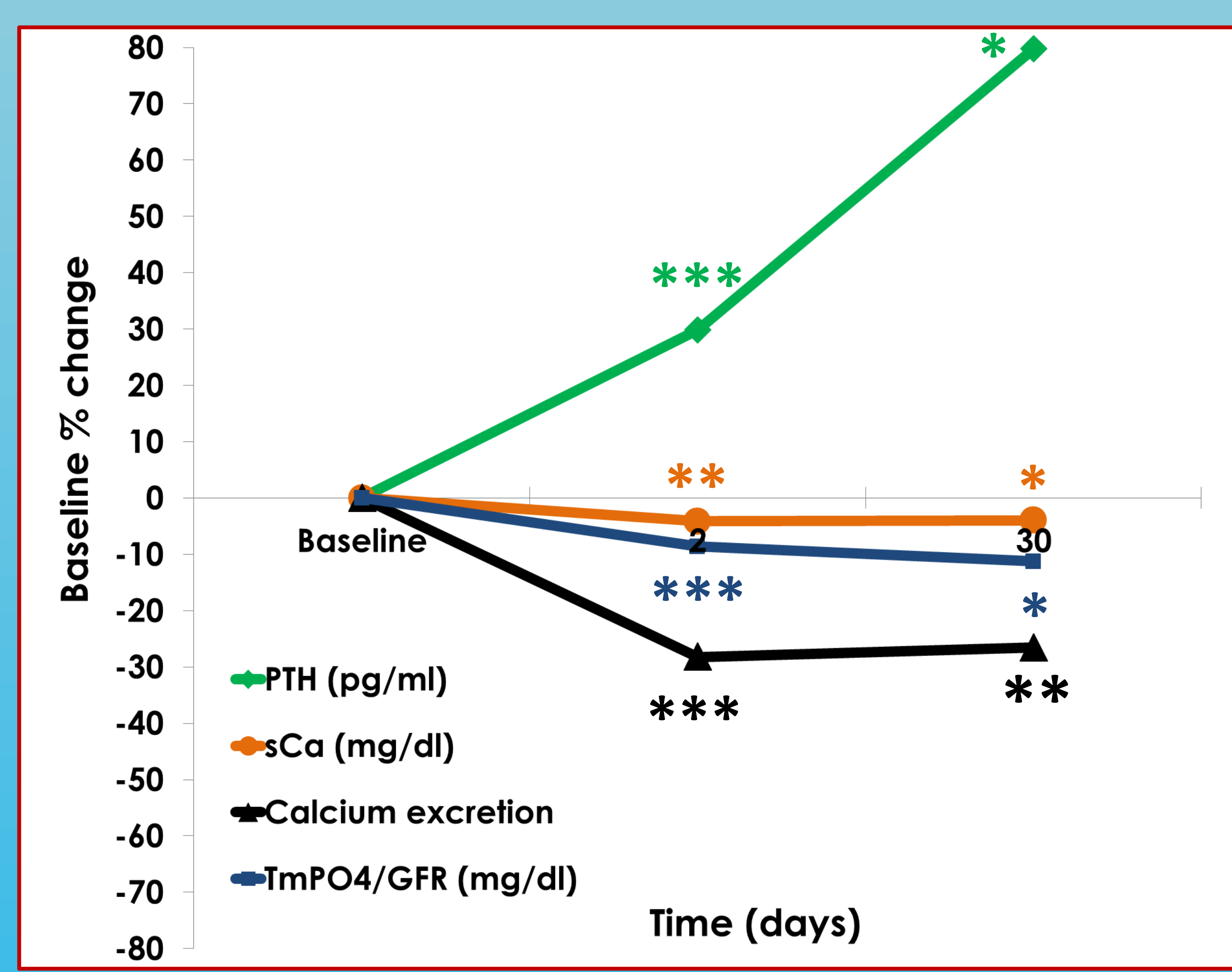

${ }^{*} p<0.001 ; " \mathrm{*} p<0.01{ }^{* * *} p<0.05$

\section{Conclusions}

Our data show that there is an acute renal damage as early as $\mathbf{2 4}$ hours after ZA infusion in osteoporotic patients with normal renal function. Renal injury is apparently reversible after 1 month.

Among the AKI biomarkers, serum MCP-1 and IL-18 have the best sensitivity in assessing the acute kidney injury. Hyperparathyroidism, secondary to reduced serum calcium levels, is responsible for reduction in $\mathrm{TmPO}_{4} / \mathrm{GFR}$ and calcium excretion. 